\section{Traumatic ribs fracture: how to treat them?}

\author{
Marco Scarci, ${ }^{1}$ Andrea Billè, ${ }^{2}$ \\ Imran Zahid, ${ }^{3}$ Tom Routledge ${ }^{2}$ \\ 'Department of Thoracic Surgery, \\ St Joseph's Healthcare, McMaster \\ University, Hamilton, Ontario, Canada; \\ 2Department of Thoracic Surgery, Guy's \\ Hospital; \\ IImperial College Medical School, London, \\ UK
}

Abstract

Flail chest complicates about $10 \%$ of patients with chest trauma and is associated with a mortality rate of $10-20 \%$ in older series, while a recent one reports no mortality. The majority of the cases are treated conservatively with internal pneumatic stabilization and pain control. In recent years, nevertheless, we assisted in the resurgence of chest wall fixation due to the availability of new devices. We report our experience in the use of mouldable titanium clips (STRACOS, Strasbourg Thoracic Osteosyntheses System; MedXpert, Heitersheim, Germany) to fix traumatic rib fracture. This device presents an advantage over previous strategies, as it is easy to apply and doesn't require drilling and screwing of the ribs.

\section{Introduction}

Flail chest complicates about $10 \%$ to $20 \%$ of patients with blunt chest trauma and is associated with a mortality rate ranging $10 \%$ to $20 \%$ in older series. ${ }^{1}$ In a more recent report, ${ }^{2}$ there was no mortality in patients with only flail chest. This condition not extensively reported. In the literature from the early nineties, the majority of cases are still treated conservatively with internal pneumatic stabilization and pain relief. ${ }^{3-5}$ Mechanical ventilation is still considered by most surgeons the standard treatment for patients with associated trauma or respiratory failure. Such strategies carry significant morbidity and mortality because of ventilatory-associated infections and increased intensive care stay. ${ }^{4}$ Moreover it does not prevent the potential chest wall deformity associated with displacement of the ribs' fractures.

The aim of our study was to report our experience in the surgical management of selected patients with flail chest using a new device for rib fixation.

\section{Materials and Methods}

In our institution, surgical management was performed in patients with flail chest in the following clinical situations: i) patients who require a thoracotomy because of associated thoracic injuries; ii) patients with deteriorating pulmonary function despite aggressive clearance of bronchial secretions and adequate analgesia; iii) patients with extensive antero-lateral flail chest and progressive dislocation of the fractured ribs, in order to prevent late chest wall deformity and a consequent restrictive disorder; iv) late fixation of chronic flail chest due to malunion of ribs' edges to prevent potential lung herniation and persistent chronic pain.

The amount of lung contusion and the clinical condition of the patient determine the timing of the intervention. In cases of extensive lung injury and mechanical ventilation, surgical fixation is unlikely to provide an immediate benefit but may contribute to reduce the overall length of stay and ventilator weaning time and the long term complications..$^{6-7}$

Patients with mild lung contusion and chest wall deformities could benefit from early surgical stabilization. In our small series, mild pulmonary contusion was defined as a sub segmental lung contusion with alveolar microhaemorrhage and normal gas exchange.

The first aim of treating chest wall trauma was to reduce the morbidity related to the lung injuries and to control the pain associated with the rib fractures. This decreases the chance of atelectasis and respiratory failure. ${ }^{8}$ Although thoracic epidural is commonly considered the gold standard for pain control, its correct placement is not always successful. In our small series we had two patients with failed epidural. Surgical stabilization also reduces the risk of long term complications, like chest wall distortions or lung herniation. ${ }^{9}$ In our experience lung herniation is a rare complication, but, several case reports from last few years describe it associated to chest trauma.

\section{Operative steps}

Surgical stabilization of flail chest is performed under general anaesthesia on single lung ventilation. The position of the patient and surgical incisions are determined by the position of the flail chest. We usually prefer a standard lateral decubitus position. This allows a better exploration of the fractured area and internal organs.

Incisions are conducted over the fractured area in a standard fashion. The surgeon must always keep in mind to maintain a balance between surgical exposure and muscle division in order to disturb as little muscle as possible to preserve respiratory function. A latissimus-sparing muscle thoracotomy was the
Correspondence: Marco Scarci, Department of Thoracic Surgery, St Joseph's Healthcare, McMaster University, 50 Charlton Avenue East L8N 4A6, Hamilton, Ontario, Canada.

Tel: +1.905.906.9131 - Fax: +1.905.540.6512.

E-mail: marco.scarci@mac.com

Key words: chest wall, titanium, artificial rib, fixation.

Received for publication: 19 June 2011. Accepted for publication: 19 September 2011.

This work is licensed under a Creative Commons Attribution NonCommercial 3.0 License (CC BYNC 3.0).

CCopyright M. Scarci et al., 2011

Licensee PAGEPress, Italy

Surgical Techniques Development 2011; 1:e20

doi:10.4081/std.2011.e20

standard approach, except in one patient with multiple posterior fractures, where a standard posterolateral thoracotomy was adopted to access the fractured area. Fractures in the anterior portion of the chest are easier to fix. While those behind the scapula (Figure 1) are more challenging because of the required division of the muscular attachments.

Fractured rib edges are exposed enough, 5 to $6 \mathrm{~cm}$, on each side to allow the clip to sit nicely. To allow better healing, when possible, we avoid ligating the intercostal vessels, these were ligated only in case of brisk bleeding. Particular care is reserved to avoid excessive exposure of the ribs. Once both ends of the ribs are exposed, they are fixed with mouldable titanium clips (STRACOS, Strasbourg Thoracic Osteosyntheses System; MedXpert, Heitersheim, Germany). These are available in 2 different lengths: 6 or 9 segments. There is a specifically designed set of instruments that allows the clip to adapt easily to the shape of the rib contour. Once ready for fixation, the clips are clipped onto the rib ends with purposely-made instruments (Figure 2).

Once a metal plate is placed, it is reinforced with a heavy nylon suture at each side if doubts exist about its anchoring strength. The clips are only about $5 \mathrm{~cm}$ in length, so extensive dissection is not necessary. The aim of the dissection should be to free enough of the rib edges to secure the device and avoid dislocation.

The pleural cavity is drained with two $28 \mathrm{Ch}$ chest tubes. The incision is closed in the usual fashion paying particular attention to avoid dislocation of the clips.

The patient is usually extubated in the operating room or transferred to intensive care for respiratory weaning. 


\section{Results}

At present we have treated 3 patients with this technique. They all presented with traumatic flail chest involving 4,6 and 6 ribs respectively and mild lung contusion on computed tomography scan.

Operative mortality was zero. Pain control was satisfactory after this procedure, transient post-operative neuralgia was observed in only one patient (5 days). One patient was managed only on paracetamol from the second postoperative day, and the other two stopped to take opioids after 7 days from surgery. They managed to mobilize effectively from the first postoperative days and engaged in physiotherapy sessions. Hospital discharge occurred between 5 and 7 days with excellent result (Figure 3).

Follow up at present has been at 7, 4 and 3 months respectively. At one-month postoperative follow-up appointment, all three patients had discontinued all painkiller medications, without complaining any chronic chest pain and they all returned to normal life. We are not aware of any further complications that may have occurred to them after discharge from the outpatient clinic.

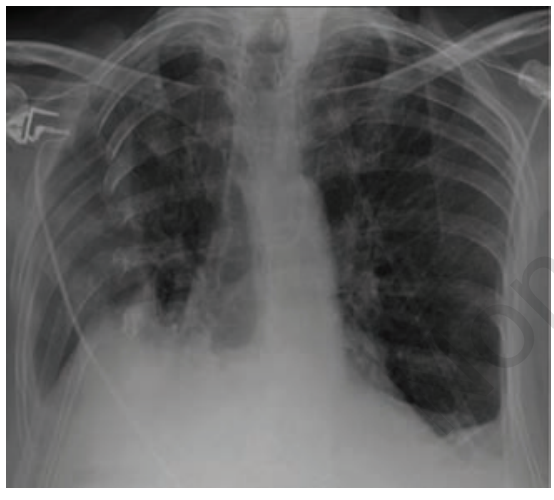

Figure 1. Chest X-ray showing a large flail chest underneath the scapula.



Figure 2. Intraoperative view with clips applied onto the ribs.

\section{Discussion}

After a chest trauma, underlying lung contusion, flail chest, pain and limited thoracic movements contribute to determine respiratory failure. Patients can also develop chronic chest pain and chest wall deformities. There is still a debate about the role of the surgical fixation of rib fractures. Lardinois et al. showed that the surgical fixation can reduce morbidity and mortality rates after chest trauma. ${ }^{10}$ Other authors, like Athanassiadi et al. believe that the surgical fixation does not improve lung function, because the respiratory insufficiency in mainly related to the lung contusions. They recommend surgical fixation in case of chest wall deformities to improve cosmetic results and in case where thoracotomy is mandatory because of threatened visceral lesions. ${ }^{11}$ In our series patients were operated only if they presented with severe chest wall deformity or visceral lesions.

To our knowledge this is the first report using the Strasbourg Thoracic Osteosyntheses Systemto fix the ribs after chest wall trauma. Other devices have been used with excellent results. ${ }^{12}$ This is a very small series with a short follow-up; nevertheless the preliminary
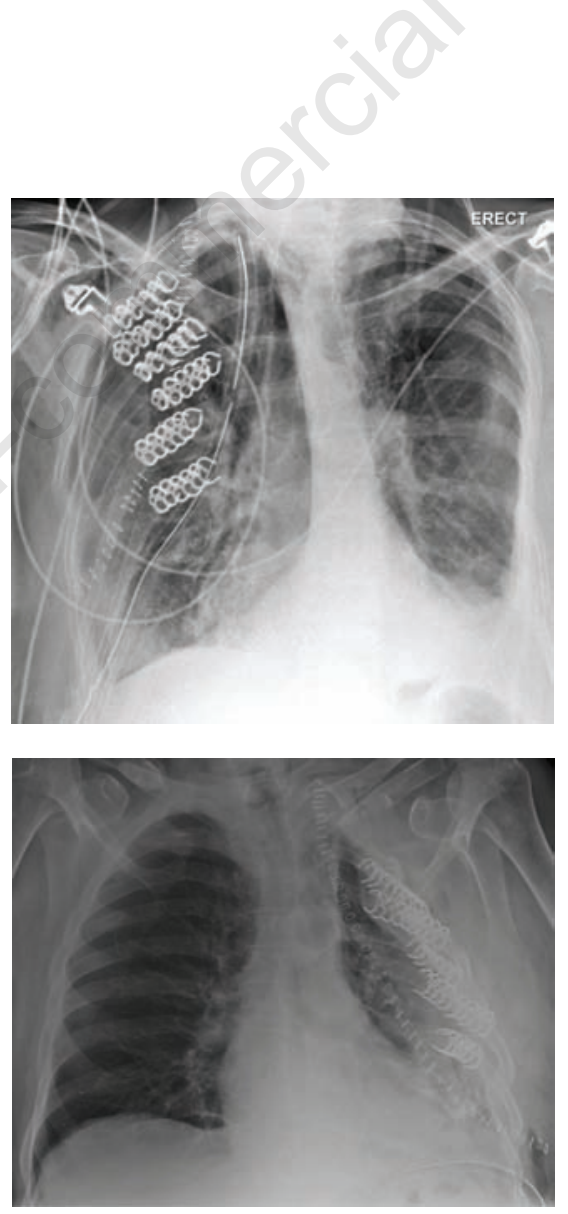

Figure 3. Chest $\mathrm{X}$-rays showing an excellent anatomical correction. results are encouraging. Our results are also supported by a recent survey where the majority of surveyed surgeons reported that rib and sternal fracture repair is indicated in selected patients; even if a much smaller proportion indicated that they had performed the procedures. ${ }^{13}$

We conclude that surgical stabilization of severe flail chest with titanium clips has been a safe and effective treatment option in selected patients in our series. The primary rational for fixation of rib fractures is to reduce early respiratory morbidity due to chest wall deformities. Restoration of the mechanics of breathing by stabilizing multiple fractures and therefore allowing the physiological buckethandle movement of the ribs contributes to this. Secondarily, there is the potential for many cases of multiple displaced rib fractures to lead to non-union or mal-union, with chronic pain and disability.

On the basis of our preliminary experience, we advocate for a trial of this device on a larger more controlled scale to validate our initial findings and assess long-term outcomes and to compare the results obtained with the other devices available.

\section{References}

1. Ciraulo DL, Elliott D, Mitchell KA, Rodriguez A. Flail chest as a marker for significant injuries. J Am Coll Surg 1994; 178:466-70.

2. Kilic D, Findikcioglu A, Akin S, et al. Factors affecting morbidity and mortality in flail chest: comparison of anterior and lateral location. Thorac Cardiovasc Surg 2011;59:45-8.

3. Davignon K, Kwo J, Bigatello LM. Pathophysiology and management of the flail chest. Minerva Anestesiol 2004;70: 193-9.

4. Gaillard M, Herne C, Mandin, Raynaud P. Mortality prognosis factors in chest injury. J Trauma 1990;30:93-6.

5. Tanaka H, Yukioka T, Yamaguti Y, et al. Surgical stabilization of internal pneumatic stabilization? A prospective randomized study of management of severe flail chest patients. J Trauma 2002;52:727-32.

6. Voggenreiter G, Neudeck F, Aufmkolk M, et al. Operative chest wall stabilization in flail chest--outcomes of patients with or without pulmonary contusion. J Am Coll Surg 1998;187:130-8.

7. Richardson JD, Adams L, Flint LM. Selective management of flail chest and pulmonary contusion. Ann Surg 1982;196: 481-7.

8. Lardinois D, Krueger T, Dusmett M, et al. Pulmonary function testing after operative 
stabilization of the chest wall for flail chest. Eur J Cardiothorac Surg 2001;20: 496-501.

9. Tzelepis GE, McCool FD, Hoppin FG Jr. Chest wall distortion in patients with flail chest. Am Rev Respir Dis 1989;140:31-7.

10. Lardinois D, Krueger T, Dusmet M, et al. Pulmonary function testing after operative stablization of the chest wall for flail chest. Eur J Cardiothoracic Surg 2001;20:496501.

11. Athanassiadi K, Thakos N, Kalantzi N, Gerazounis M. Prognostic factors in flail chest patients. Eur J Cardiothoracic Surg 2010;38:466-471.

12. Mayberry JC, Terhes JT, Ellis TJ, et al.
Absorbable plates for rib fracture repair: preliminary experience. J Trauma 2003;55: 835-9.

13. Mayberry JC, Ham LB, Schipper PH, et al. Surveyed opinion of American trauma, orthopedic, and thoracic surgeons on rib and sternal fracture repair. J Trauma 2009; 66:875-9. 